\title{
Salvage therapy with temozolomide for recurrent or progressive high-grade gliomas refractory to $\mathrm{ACNU}$ [1-(4-amino-2-methyl-5-pyrimidynyl) methyl-3- (2-chloroethyl)-3-nitrosourea hydrochloride]
}

\author{
MIZUHIKO TERASAKI, TAKASHI TOKUTOMI and MINORU SHIGEMORI \\ Department of Neurosurgery, Kurume University School of Medicine, Fukuoka 830-0011, Japan
}

Received December 5, 2008; Accepted February 5, 2009

DOI: 10.3892/mmr_00000115

\begin{abstract}
This study aimed to determine safety, response rate, toxicity and 6-month progression-free survival (PFS) by using temozolomide (TMZ) as salvage chemotherapy for 25 adults with recurrent or progressive high-grade gliomas (HGGs) who failed 1-(4-amino-2-methyl-5-pyrimidynyl) methyl-3(2-chloroethyl)-3-nitrosourea hydrochloride (ACNU) therapy. Twenty-six patients with recurrent or progressive ACNU refractory HGG, including 12 with glioblastoma (GBM) and 13 with anaplastic astrocytoma (AA) were evaluated in a prospective study of temozolomide salvage chemotherapy. Following maximal surgical resection, the patients received $2-4$ cycles of procarbazine $\left(100 \mathrm{mg} / \mathrm{m}^{2}\right.$, days $\left.1-5\right), \mathrm{ACNU}$ $\left(80 \mathrm{mg} / \mathrm{m}^{2} / \mathrm{d}^{1}\right.$, day 5$)$ and, on days 5 and 12 , cepharantine (70 mg) and vincristine $\left(1.4 \mathrm{mg} / \mathrm{m}^{2}\right)$. TMZ $\left(150-200 \mathrm{mg} / \mathrm{m}^{2} / \mathrm{d}\right.$, days 1-5) was also administered every 28 days for $\leq 24$ cycles. The six-month PFS was 50\% (mean 10 months; 95\% CI, 7-14 months) in 12 GBM patients and 39\% (mean 17 months; 95\% CI, 7-28 months) in 13 patients with AA. The best response to chemotherapy had no impact on the duration of disease control. Treatment-related toxicities included infections, while two (8\%) patients developed neutropenia. In conclusion, TMZ can benefit patients with ACNU refractory HGG.
\end{abstract}

\section{Introduction}

Temozolomide (TMZ; Temodar ${ }^{\circledR}$, Temodal ${ }^{\circledR}$; Schering-Plough Corp., Kenilworth, NJ) has replaced nitrosourea as the current standard for the initial treatment of high-grade gliomas (HGGs) (1). However, no standard therapy for recurrent or progressive HGGs currently exists. Efforts to manage patients with recurrent disease are hampered by histological complexity,

Correspondence to: Dr Mizuhiko Terasaki, Department of Neurosurgery, Kurume University School of Medicine, 67 Asahimachi, Kurume, Fukuoka 830-0011, Japan

E-mail: jintara@med.kurume-u.ac.jp

Key words: ACNU, temozolomide, refractory tumor resistance to radiotherapy and chemotherapy, local dissemination of the tumor beyond radiotherapy fields, and the inability of chemotherapy to penetrate the blood-brain barrier $(1,2)$. Consequently, treatment with conventional chemotherapy has been ineffective (1). More innovative approaches, such as high-dose chemotherapy with stem-cell transplantation or stereotactic radiosurgery, are only moderately successful (3). Patients with secondary HGGs or with lesions that transformed from a low to a higher grade also fare poorly (2). Repeat surgery to debulk a recurrent tumor can have favorable, though short-term, survival benefits for some patients (4). Recent studies have combined molecular-targeted therapy with other chemotherapy agents. Among these combinations, bevacizumab with irinotecan is effective (5). However, further salvage therapy after relapse is not yet available.

Despite initial enthusiasm for the use of TMZ alone in adults with brain tumors, a Phase II study showed that only 5-35\% of adults with relapsed or progressive HGGs responded to its application, though TMZ did appear to have an advantage over other alkylating agents (e.g., carmustine; BCNU) for tumors refractory to nitrosoureas (1). The impact of TMZ treatment for nitrosourea-refractory HGG on survival, response, duration of improvement and disease stability is unknown. Further studies comparing the survival benefit of TMZ with previously used nitrosoureas are required to determine which salvage therapy is most effective. Regarding toxicity, we know that combination therapy with nitrosourea causes more hematological toxicity than TMZ therapy, but has the same impact on survival in elderly patients with glioblastoma (GBM) as TMZ (6).

In this study, we evaluated early outcome data from a pilot study of TMZ therapy for patients with recurrent or progressive HGG refractory to 1-(4-amino-2-methyl-5-pyrimidynyl) methyl-3-(2-chloroethyl)-3-nitrosourea hydrochloride (ACNU) with respect to feasibility, survival, rate of response and toxicity.

\section{Materials and methods}

This study was conducted between August 2005 and January 2008. The protocols were were determined to have minimal risk and approved by each local institutional ethics committee of Kurume University. 
Patients. All patients had recurrent or progressive HGG, consisting of anaplastic astrocytoma (AA) or GBM, after failed nitrosourea therapy. We confirmed anaplastic lesions histologically and brainstem lesions based on neuroradiographic findings, although biopsy was strongly recommended for these patients. Disease progression was confirmed by magnetic resonance imaging (MRI), which radiographically documented measurable residual disease. Patients received MRI scans with galodinium to reveal the amount of residual disease present prior to treatment. Before the initiation of therapy, patients were required to have absolute neutrophil counts of $>1.000 / \mathrm{mm}^{3}$, platelet counts of $>100.000 / \mathrm{mm}^{3}$, and normal liver and renal functions. Patients also underwent spinal MRI and a cytological examination of their cerebrospinal fluid (CSF).

Treatment regimen. Patients received the maximal surgical resection possible, followed by chemoradiotherapy as initial therapy. Chemotherapy was administered for 12 consecutive days according to the following protocol: procarbazine, $100 \mathrm{mg} / \mathrm{m}^{2}$, days $1-5$; ACNU, $80 \mathrm{mg} / \mathrm{m}^{2} / \mathrm{d}^{1}$, day 5 ; cepharantine, $70 \mathrm{mg}$, days 5 and 12 and vincristine, $1.4 \mathrm{mg} / \mathrm{m}^{2}$, days 5 and 12. Patients received procarbazine to deplete $\mathrm{O}^{6}$ methylguanine-DNA methyltransferase and maximize the effect of ACNU (7). For tumor cells overcoming metaphase in the cell cycle, two cycles of cepharantine were administered at 28-day intervals to inhibit p-glycoprotein and enhance the effects of vincristine (8). Radiotherapy was synchronously administered. Maintenance chemotherapy consisted of the same regimen. Further cycles were administered at 28-day intervals.

Radiotherapy consisted of 60 Gy in 30 fractions to the tumor area, determined from T2WI MRIs, and an additional 2 -cm margin. After chemotherapy was completed, we reevaluated the extent of the disease to determine the presence of residual tumor sites. Patients with radiographic evidence of localized residual tumors were strongly considered for maintenance chemotherapy, while patients who showed radiographic evidence of tumor progression at any time during or after induction chemotherapy were not eligible for maintenance chemotherapy, and were considered for salvage chemotherapy with TMZ.

Patients on salvage therapy received TMZ at 150-200 mg/ $\mathrm{m}^{2}$ /day on days 1-5 with antiemetic medication. Cycles were repeated every 28 days for up to 24 cycles.

Supportive care. Platelet counts were maintained above $10.000 / \mathrm{mm}^{3}$ and hemoglobin counts above $8.0 \mathrm{~g} / \mathrm{dl}$ with transfusions as necessary, unless higher parameters were clinically indicated; i.e., acute hemorrhage or respiratory distress. Blood products were irradiated. Febrile neutropenic patients were treated with broad-spectrum intravenous antibiotics and antifungal agents when appropriate.

Patient monitoring/toxicity criteria. Our protocol required frequent complete blood count and biochemical studies throughout the course of chemotherapy. The National Institutes of Health Common Toxicity Criteria 2 were used to grade toxicity. At prescribed times during and following chemotherapy, the disease status of the patients was monitored with the appropriate neurological examinations, CSF cytologic examinations and neuroimaging studies to assess the effect of chemotherapy on post-operative residual tumors. MRIs were obtained after every 2 cycles.

Evaluation. Our neuroradiological criteria for response or relapse were: complete response (CR), complete resolution of the tumor on enhanced MRI scan; partial response (PR), a $>50 \%$ reduction in the product of the greatest tumor diameter and its perpendicular diameter as measured on an enhanced MRI scan; minor response (M), a 25-50\% reduction in the product of the greatest tumor diameter and its perpendicular diameter; stable disease (SD), a $<25 \%$ decrease in the product of the perpendicular diameters described; progressive disease (PD), a $>25 \%$ increase in tumor size, or appearance of the tumor in a previously uninvolved area.

Investigators at the treating institution determined the level of relapse or response to treatment. We performed no central neuroradiographic review. Pathologic findings were determined at the treating institution and classified according to World Health Organization Criteria.

Informed consent. Signed informed consent was obtained from all patients. Potential risks and benefits, therapeutic alternatives such as conventional chemotherapy and irradiation, and supportive care were discussed.

Statistical considerations. Our primary end-point for this study was determining progression-free survival (PFS). Overall survival, rate of response and safety in the intent-to-treat population were also assessed. Kaplan-Meier analysis was used to estimate the distribution of PFS. PFS was calculated from the date of the initiation of TMZ chemotherapy to the date of radiographic evidence of disease progression. Survival was calculated from the initiation of TMZ chemotherapy to the date of the last follow-up or the date of death due to any causes. The cut-off for this analysis was January 2008.

\section{Results}

Demographics. Twenty-five patients were treated with TMZ for recurrent or progressive high-grade gliomas. Table I summarizes the patient demographics. The median age of the 18 male and 7 female patients at the time of treatment was 51 years (range 17-70). Diagnoses at study entry included 13 patients with AA ( 2 with secondary and 11 with progressive disease) and 12 patients with recurrent GBM. All patients had received prior radiotherapy and chemotherapy. Fourteen were treated at first recurrence; the remainder, at second or further recurrence. Table II lists the data for each patient.

Outcome. Of the 25 patients, 5 had PR; 17 SD, and 3 PD. The duration of response in the 4 AA patients with PR was $3+, 4,22+$ and 41+ months. Only one GBM patient had PR (response rate 7\%). The responses of the patient are summarized in Table III.

To date, 6 patients with AA and 11 with GBM had disease progression, of which $3(50 \%)$ and $10(91 \%)$, respectively, succumbed to the disease. The sites of progression were local (10 patients) or disseminated (3 patients). Time-to-relapse 
Table I. Demographics of 25 patients with recurrent or progressive high-grade gliomas enrolled in a pilot study.

\begin{tabular}{lc}
\hline Characteristic & No. of patients \\
\hline Male/female & $18 / 7$ \\
Age at diagnosis (years) & \\
$\quad$ Median & 51 \\
$\quad$ Range & $17-70$ \\
Nature of recurrence & \\
$\quad$ Recurrence & 12 \\
$\quad$ Treatment-induced & 13 \\
\hline
\end{tabular}

ranged from 2 to 12 months post-treatment (median 5.8 months). Ten patients with AA and 2 with GBM are alive with disease. After a median follow-up of 49 months, the 6-month PFS for AA patients was 39\% [mean 17 months; 95\% confidence interval (CI), 7-28 months] and for GBM patients 50\% (mean 10 months; 95\% CI, 7-14 months). Median survival time from the initiation of TMZ chemotherapy after ACNU failure was 57 months for AA patients and 17 months for GBM patients.

Toxicity. In general, treatment was relatively well tolerated. Toxicity during the 154 courses of therapy was mild. Two patients $(8 \%)$ developed grade 4 neutropenia, and two had

Table II. Patient characteristics, diagnosis and prior therapy.

\begin{tabular}{|c|c|c|c|c|c|c|}
\hline $\begin{array}{l}\text { No. of } \\
\text { patients }\end{array}$ & $\begin{array}{c}\text { Age } \\
\text { (years) }\end{array}$ & $\begin{array}{c}\text { Initial } \\
\text { diagnosis }\end{array}$ & Prior therapy & $\begin{array}{l}\text { No. of } \\
\text { recurrences }\end{array}$ & $\begin{array}{l}\text { Nature of } \\
\text { recurrence }\end{array}$ & $\begin{array}{l}\text { Time from initial } \\
\text { diagnosis (years) }\end{array}$ \\
\hline 1 & 42 & AA & RT/ACNU & 5 & Progression & 18.4 \\
\hline 2 & 54 & AA & RT/ACNU/VCR & 4 & Progression & 10.3 \\
\hline 3 & 37 & AA & RT/ACNU/VCR & 1 & Progression & 3.3 \\
\hline 4 & 20 & AA & $\begin{array}{l}\text { (1) } \mathrm{RT} / \mathrm{CBDCA} / \mathrm{VP} 16 \\
\text { (2) ACNU }\end{array}$ & 3 & Progression & 2.0 \\
\hline 5 & 49 & LGA & $\begin{array}{l}\text { (1) } \mathrm{RT} \\
\text { (2) ACNU }\end{array}$ & 2 & Progression & 5.3 \\
\hline 6 & 57 & GBM & $\mathrm{RT} / \mathrm{ACNU}$ & 2 & Progression & 2.3 \\
\hline 7 & 43 & LGA & $\begin{array}{l}\text { (1) RT/ACNU/VCR } \\
\text { (2) Repeat surgery }\end{array}$ & 1 & Progression & 2.3 \\
\hline 8 & 55 & AA & $\mathrm{RT} / \mathrm{ACNU}$ & 1 & Progression & 0.7 \\
\hline 9 & 69 & GBM & RT/ACNU/VCR & 3 & Progression & 1.8 \\
\hline 10 & 51 & AA & RT/ACNU & 1 & Progression & 0.6 \\
\hline 11 & 50 & GBM & RT/ACNU/VCR & 1 & Recurrence & 0.3 \\
\hline 12 & 57 & GBM & RT/ACNU & 1 & Recurrence & 0.4 \\
\hline 13 & 73 & AA & RT/ACNU & 1 & Progression & 2.0 \\
\hline 14 & 68 & GBM & RT/ACNU/VCR & 1 & Recurrence & 1.6 \\
\hline 15 & 69 & GBM & RT/ACNU/VCR & 1 & Recurrence & 0.3 \\
\hline 16 & 70 & AA & RT/ACNU/VCR & 1 & Recurrence & 0.5 \\
\hline 17 & 47 & AA & $\begin{array}{l}\text { (1) RT/ACNU/VCR } \\
\text { (2) PAV } \\
\text { (3) Repeat surgery }\end{array}$ & 3 & Recurrence & 4.3 \\
\hline 18 & 40 & AA & $\begin{array}{l}\text { (1) RT/ACNU/VCR } \\
\text { (2) Re-RT }\end{array}$ & 2 & Progression & 11.3 \\
\hline 19 & 38 & GBM & RT/ACNU/VCR & 1 & Recurrence & 0.6 \\
\hline 20 & 41 & GBM & $\begin{array}{l}\text { (1) RT/ACNU/VCR } \\
\text { (2) Repeat surgery }\end{array}$ & 2 & Recurrence & 0.8 \\
\hline 21 & 67 & GBM & RT/ACNU/VCR & 1 & Recurrence & 0.3 \\
\hline 22 & 55 & GBM & $\begin{array}{l}\text { (1) RT/ACNU/VCR } \\
\text { (2) Repeat surgery }\end{array}$ & 2 & Recurrence & 0.8 \\
\hline 23 & 17 & GBM & $\begin{array}{l}\text { (1) RT/ACNU/VCR } \\
\text { (2) CBDCA/VP16 }\end{array}$ & 2 & Progression & 1.7 \\
\hline 24 & 52 & GBM & RT/ACNU/VCR & 1 & Recurrence & 0.8 \\
\hline 25 & 26 & AA & RT/ACNU/VCR & 1 & Recurrence & 4.3 \\
\hline
\end{tabular}

AA, anaplastic astrocytoma; ACNU, nimustine hydrochloride; GBM, glioblastoma; CBDCA, carboplatin; PAV, procarbazine + nimustine hydrochloride + vincristine combination chemotherapy; RT, radiotherapy; VCR, vincristine. 
Table III. Response and outcome for the 25 patients.

\begin{tabular}{|c|c|c|c|c|c|c|}
\hline $\begin{array}{l}\text { No. of } \\
\text { patients }\end{array}$ & $\begin{array}{l}\text { Diagnosis at } \\
\text { recurrence }\end{array}$ & $\begin{array}{l}\text { Histological confirmation } \\
\text { at initial/recurrence }\end{array}$ & Total cycles & Best response & $\begin{array}{c}\text { PFS } \\
\text { (months) }\end{array}$ & Outcome \\
\hline 1 & AA & Yes/Yes & 2 & SD & $2+$ & AWD, 235+mo. \\
\hline 2 & AA & Yes/Yes & 5 & SD & $3+$ & AWD, 126+mo. \\
\hline 3 & AA & Yes/No & 15 & SD & 20 & DOD, 29 mo. \\
\hline 4 & AA & Yes/No & 17 & PR & $41+$ & AWD, 64+mo. \\
\hline 5 & AA & $\mathrm{Yes} / \mathrm{No}$ & 5 & PR & $3+$ & AWD, 66+mo. \\
\hline 6 & GBM & Yes/Yes & 17 & SD & $17+$ & AWD, 44+mo \\
\hline 7 & $\mathrm{AA}$ & Yes/Yes & 2 & PD & 4 & AWD, 40+mo. \\
\hline 8 & AA & Yes/Yes & 3 & SD & $28+$ & AWD, 36+mo \\
\hline 9 & GBM & Yes/No & 1 & PD & 5 & DOD, 26 mo. \\
\hline 10 & $\mathrm{AA}$ & Yes/No & 23 & PR & $22+$ & AWD, 29+mo. \\
\hline 11 & GBM & Yes/No & 6 & SD & 5 & AWD, 22+mo. \\
\hline 12 & GBM & Yes/Yes & 7 & SD & 6 & DOD, 16 mo. \\
\hline 13 & $\mathrm{AA}$ & Yes/No & 1 & PD & 1 & AWD, 38+mo. \\
\hline 14 & GBM & Yes/Yes & 4 & SD & 5 & DOD, 15 mo. \\
\hline 15 & GBM & Yes/No & 9 & $\mathrm{SD}$ & 10 & DOD, 13 mo. \\
\hline 16 & $\mathrm{AA}$ & Yes/Yes & 4 & SD & 4 & AWD, 27+mo. \\
\hline 17 & $\mathrm{AA}$ & Yes/Yes & 1 & SD & 1 & DOD, $57 \mathrm{mo}$. \\
\hline 18 & $\mathrm{AA}$ & Yes/No & 7 & SD & $28+$ & AWD, 160+mo. \\
\hline 19 & GBM & Yes/No & 3 & PR & 6 & DOD, 19 mo. \\
\hline 20 & GBM & Yes/Yes & 2 & SD & 4 & DOD, 17 mo. \\
\hline 21 & GBM & Yes/No & 3 & SD & 5 & DOD, 8 mo. \\
\hline 22 & GBM & Yes/Yes & 4 & SD & 4 & DOD, 15 mo. \\
\hline 23 & GBM & Yes/No & 6 & SD & 7 & DOD, 20 mo. \\
\hline 24 & GBM & Yes/No & 3 & SD & 3 & DOD, 17 mo. \\
\hline 25 & $\mathrm{AA}$ & Yes/No & 4 & PR & 4 & DOD, 63 mo. \\
\hline
\end{tabular}

AA, anaplastic astrocytoma; AWD, alive with disease; DOD, died of disease; GBM, glioblastoma; PFS, progression-free survival; PD, progressive disease; $\mathrm{PR}$, partial response; $\mathrm{SD}$, stable disease.

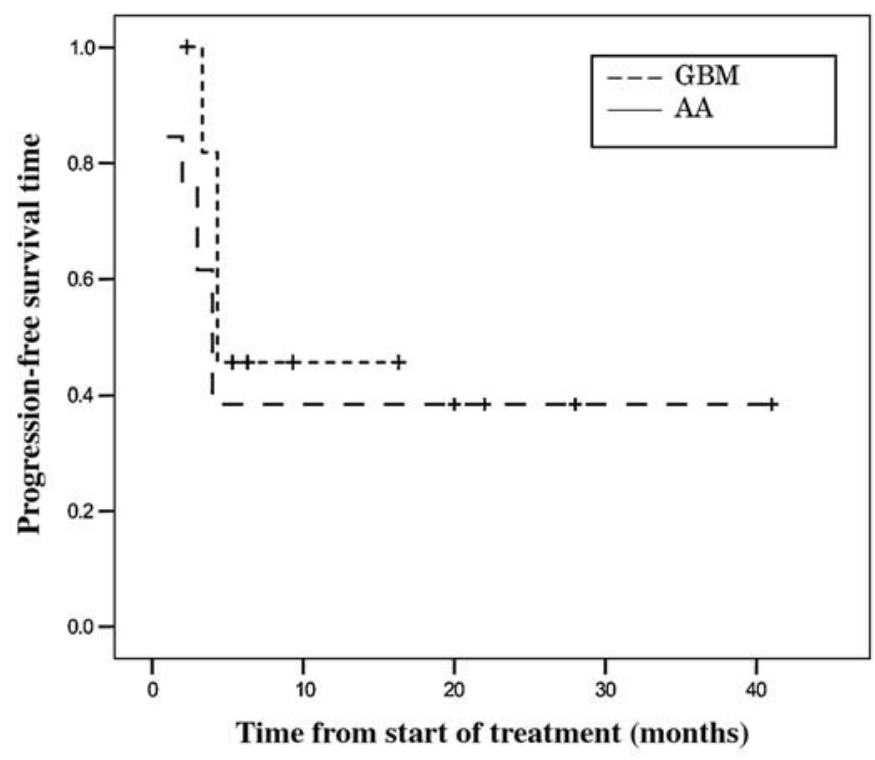

Figure 1. Progression-free survival vs. time from start of treatment for GBM and AA patients. grade 3 and 4 thrombocytopenia. One of these latter two received a transfusion of 3 units of platelets. Prolonged thrombocytopenia or neurotoxicity occasionally delayed treatment. However, no patients were withdrawn from the study due to hematological toxicity, nephrotoxicity or neurotoxicity. None reported infection and no patient was hospitalized for TMZ side effects.

\section{Discussion}

Adjuvant chemotherapy provides a modest survival advantage for patients with malignant gliomas (9). Nitrosourea-based adjuvant chemotherapy used to be regarded as the most active regimen for HGGs. However, the current standard has shifted to TMZ (10). For patients with recurrent or progressive HGG treated with TMZ, chemotherapy as an adjuvant has been widely investigated, but has shown only limited success (1). Recent studies focusing on combining molecular-targeted therapy with other chemotherapy agents, including bevacizumab and irinotecan, have yielded excellent results (5). However, further salvage after relapse still requires study. In 
the search for a single-agent treatment with improved efficacy, safety and quality of life, TMZ has shown activity in recurrent or progressive HGG (11). However, no conclusive randomized comparisons between nitrosourea- and TMZ-based therapies have been published. Wolff and coworkers (12) analyzed survival gain from 504 cohorts totaling 24,193 patients treated with various nitrosoureas, and compared the results against TMZ. They reported that ACNU- or lomustinecontaining regimens were superior to BCNU-containing regimens for patients with HGG. We found that combination therapy with nitrosourea has more hematological toxicity than TMZ therapy, but has the same impact on survival as TMZ (6). The present study demonstrates that TMZ salvage therapy has activity in patients with recurrent or progressive nitrosourea-refractory HGG. Our study included patients with GBM and AA. A clinically significant 6-month PFS of $50 \%$ with mild toxicity and good quality of life was found for patients with GBM. We conclude that salvage treatment with TMZ, for those who received nitrosourea-based agents as initial therapy, has potential activity, ease of administration and mild toxicity.

In single-agent trials of TMZ for adults with recurrent brain tumors, response rates were low. Only 5\% of adults with recurrent GBM responded to TMZ $(11,13)$. Adults with recurrent $\mathrm{AA}$ and anaplastic oligoastrocytoma had a response rate of $35 \%$; however, their responses were generally shortterm and PFS at 6 months was $46 \%$ (14). Furthermore, $60 \%$ of the AA and anaplastic oligoastrocytoma cases had received prior chemotherapy that included nitrosourea (14). Although the patients in our study had received prior ACNU chemotherapy, and therefore may have had chemoresistant tumors, our data are consistent with the previously obtained results. Though the mechanisms of resistance are similar in nitrosoureas and TMZ (1,7), a number of patients who progressed after ACNU chemotherapy responded to TMZ. Pre-clinical data showed that TMZ demonstrated broad spectrum and schedule-dependent antitumor activity with relatively little toxicity, but we cannot conclude that a standard regimen of TMZ for ACNU refractory patients resulted in response. The subsequent disease control observed in 6 of 25 patients suggests that the activity of this drug relates more to its pharmacodynamics than to ACNU resistance.

The lack of toxicity in this trial could be due partly to selection bias. This was a pilot study, and clinicians may have selected patients with good performance status who managed intensive chemotherapy. Nonetheless, some of these patients had been heavily pre-treated (including one with multi-drug combination chemotherapy). Despite prior treatment, most patients had only grade $0-1$ hematotoxicity. They reported no nausea or vomiting. The only other toxicity reported was grade 2 constipation in 5 patients. In recent Phase II studies on TMZ for adults with recurrent HGG, toxicity was much lower, including 4 patients (4\%) with neutropenia and $8(7 \%)$ with thrombocytopenia (14). This may be have been due to differences in patient characteristics; in particular, their prior chemotherapy regimens which were single-agent procarbazine or dacarbazine.

Notably, four patients in our series had repeat surgery. The activity of this combination (repeat surgery and TMZ) in GBM has already been noted in a previous report (15).
Hence, the findings in this study may not be generalized to more typical recurrences of HGGs. Of note, 9 patients with primary GBM $(75 \%)$ experienced disease stabilization for 5 months (range 5-17).

This pilot study confirms the promising activity of TMZ for recurrent or progressive nitrosourea-refractory HGGs. This benefit is not only limited to patients who fail nitrosourea, but may also allow for a broader range of alternatives with molecular-targeted therapies for salvage regimens after the initial treatment failure of ACNU. Ease of administration, lack of toxicity and impressive responses in this small group of patients with malignant brain tumors make this protocol attractive for further randomized studies. The efficacy of more intensive TMZ regimens, such as ' 1 week-on/1 week-off' or '3 weeks-on/1 week-off' schedules, should also be investigated in this setting.

\section{References}

1. Stupp R, Hegi ME, van den Bent MJ, et al: Changing paradigms an update on the multidisciplinary management of malignant glioma. Oncologist 11: 165-180, 2006.

2. Kleihues P and Cavenee WK (eds): Pathology and genetics. In: Tumours of the Nervous System. IARC Press, Lyon, pp9-54, 2000.

3. Butowski NA, Sneed PK and Chang SM: Diagnosis and treatment of recurrent high-grade astrocytoma. J Clin Oncol 24: 1273-1280, 2006.

4. Guyotat J, Signorelli F, Frappaz D, Madarassy G, Ricci AC and Bret P: Is reoperation for recurrence of glioblastoma justified? Oncol Rep 7: 899-904, 2000.

5. Vredenburgh JJ, Desjardins A, Herndon JE II, et al: Bevacizumab plus irinotecan in recurrent glioblastoma multiforme. J Clin Oncol 25: 4722-4729, 2007.

6. Terasaki M, Abe T, Miyagi N, Ogo E and Shigemori M: Feasibility and response to 1-(4-amino-2-methyl-5-pyrimidynyl) methyl-3-(2-chloroethyl)-3-nitrosourea hydrochloride chemotherapy with pre-treated procarbazine for elderly patients with newly diagnosed glioblastoma. J Neurooncol 81: 265-269, 2007.

7. Valavanis C, Souliotis VL and Kyrtopoulos SA: Differential effects of procarbazine and methylnitrosourea on the accumulation of $\mathrm{O}^{6}$-methylguanine and the depletion and recovery of $\mathrm{O}^{6}$-alkylguanine-DNA alkyltransferase in rat tissues. Carcinogenesis 15: 1681-1688, 1994.

8. Hirai M, Tanaka K, Shimizu T, et al: Cepharantin, a multidrug resistant modifier, is a substrate for p-glycoprotein. J Pharmacol Exp Ther 275: 73-78, 1995.

9. Fine HA, Dear KB, Loeffler JS, Black PM and Canellos GP: Meta-analysis of radiation therapy with and without adjuvant chemotherapy for malignant glioma in adults. Cancer 71: 2585-2597, 1993.

10. Stupp R, Mason WP, van den Bent MJ, et al: Radiotherapy plus concomitant and adjuvant temozolomide for glioblastoma. $\mathrm{N}$ Engl J Med 352: 987-996, 2005.

11. Yung WK, Albright RE, Olson J, et al: A phase II study of temozolomide vs. procarbazine in patients with glioblastoma multiforme at first relapse. Br J Cancer 83: 588-593, 2000.

12. Wolff JE, Berrak S, Koontz Webb SE and Zhang M: Nitrosourea efficacy in high-grade glioma: a survival gain analysis summarizing 504 cohorts with 24193 patients. J Neurooncol 88: 57-63, 2008.

13. Wick W and Weller M: How lymphotoxic is dose-intensified temozolomide? The glioblastoma experience. J Clin Oncol 23: 4235-4236, 2005.

14. Yung WK, Prados MD, Yaya-Tur R, et al: Multicenter phase II trial of temozolomide in patients with anaplastic astrocytoma or anaplastic oligoastrocytoma at first relapse. Temodal Brain Tumor Group. J Clin Oncol 17: 2762-2771, 1999.

15. Terasaki M, Ogo E, Fukushima S, et al: Impact of combination therapy with repeat surgery and temozolomide for recurrent or progressive glioblastoma multiforme: a prospective trial. Surg Neurol 68: 250-254, 2007. 